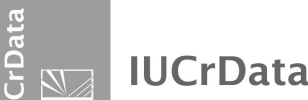

ISSN 2414-3146

Received 25 August 2017

Accepted 25 August 2017

Edited by M. Bolte, Goethe-Universität Frankfurt, Germany

Keywords: crystal structure; centrosymmetric dimer; layer structure; organochlorine compound; alkyne.

CCDC reference: 1570830

Structural data: full structural data are available from iucrdata.iucr.org

\section{rac-1,1,1,6,6,6-Hexachlorohex-3-yne-2,5-diol hemihydrate}

\author{
Heiner Detert* and Dieter Schollmeyer
}

University of Mainz, Institut of Organic Chemistry, Duesbergweg 10-14, 55099 Mainz, Germany. *Correspondence e-mail: detert@uni-mainz.de

The asymmetric unit of the title compound, $\mathrm{C}_{6} \mathrm{H}_{4} \mathrm{Cl}_{6} \mathrm{O}_{2} \cdot 0.5 \mathrm{H}_{2} \mathrm{O}$, contains one molecule of 1,1,1,6,6,6-hexachlorohex-3-yne-2,5-diol and half a water molecule located on a twofold rotation axis. In the crystal, pairs of hexachlorohexynediol molecules form centrosymmetric dimers connected via pairwise $\mathrm{O}-\mathrm{H} \cdots \mathrm{O}$ hydrogen bonds. These dimers are connected by water molecules, resulting in layers parallel to the $a b$ plane.
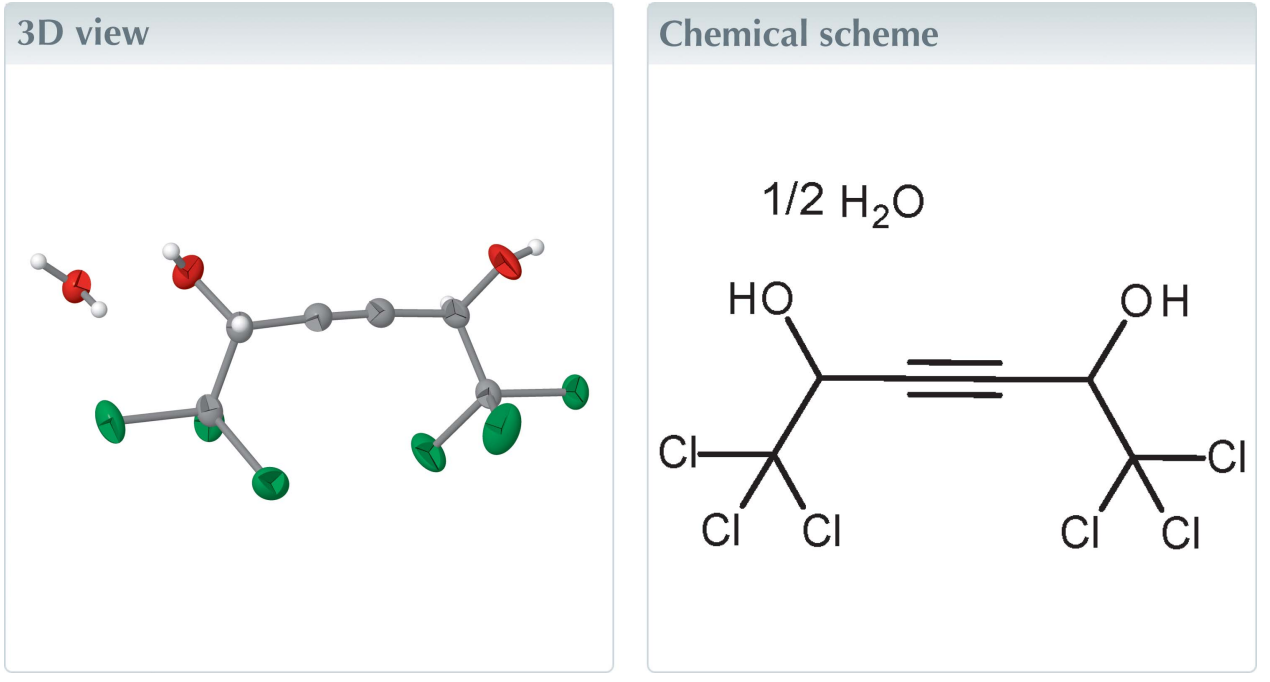

\section{Structure description}

Highly chlorinated compounds are of current interest because they are intermediates in the formation of environmental pollutants (Taylor et al., 2000) and they are useful as chemical substrates (Rahimi et al., 2009; Schmidt et al., 2009). Furthermore, their rearrangements (McIntosh et al., 2014; Schollmeyer \& Detert, 2017; Detert et al., 2009) are a topic in its own right. The monoclinic unit cell contains four centrosymmetric dimers composed of one molecule with an $R, R$-configuration, one with an $S, S$-configuration and four water molecules, the latter is located on a twofold rotation axis.

In the monoclinic crystal, the hexachlorohexynediol molecules adopt a gauche conformation $\left[\mathrm{C} 1-\mathrm{C} 2 \cdots \mathrm{C} 5-\mathrm{C} 6=30.4(2)^{\circ}\right]$ with a nonperfect $C 2$ symmetry (Fig. 1). The $\mathrm{C}-\mathrm{Cl}$ bonds of the trichloromethyl groups vary between $1.756(3)(\mathrm{C} 6-\mathrm{Cl} 4)$ and 1.776 (3) $\AA$ ( C1 - Cl2). With bond angles of 176.9 (3) and 175.8 (3) and a torsion angle of $3(10)^{\circ}$, the alkyne unit is not perfectly linear. An $R, R$ - and an $S, S$-configured diole are connected via short hydrogen bonds $\left[\mathrm{O} 1-\mathrm{H} 1 O \cdots \mathrm{O} 2^{\mathrm{ii}}=2.725(3) \AA\right]$ to a centrosymmetric dimer (Table 1). A C $-\mathrm{H} \cdots$ O hydrogen bond $\left[\mathrm{C} 5-\mathrm{H} 5 \cdots \mathrm{O} 1^{\mathrm{i}}=3.297\right.$ (4) $\AA$ ] forms a chain parallel to the $b$ axis. Hydrogen bonds between atoms $\mathrm{O} 1$ and $\mathrm{O} 2$ to the water molecule $\left[\mathrm{O} 2-\mathrm{H} 2 \mathrm{O} \cdots \mathrm{O} 3^{\mathrm{i}}=2.773(3) \AA\right.$ and $\mathrm{O} 3-\mathrm{H} 3 O \cdots \mathrm{O} 1=2.999$ (3) $\left.\mathrm{A}\right]$ connect these chains into layers in the $a b$ plane (Fig. 2). 


\section{Synthesis and crystallization}

The title compound was prepared from ethyl magnesium bromide, acetylene and chloral according to Gorgues et al. (1986) and Dupont (1910) followed by aqueous work-up. A mixture of three stereoisomers was obtained. Recrystallization from ethanol solution gave the title compound. ${ }^{1} \mathrm{H}$ NMR: $\left(\mathrm{CDCl}_{3} / \mathrm{DMSO}-d_{6}, 400 \mathrm{MHz}\right): \delta 7.05(2 \mathrm{H}, \mathrm{OH}), 4.79(2 \mathrm{H}, \mathrm{CH}$, $\left.{ }^{1} J_{\mathrm{CH}}=154 \mathrm{~Hz}\right)$. Recrystallization from chloroform solution yielded colourless crystals (m.p. $408 \mathrm{~K}$ ).

\section{Refinement}

Crystal data, data collection and structure refinement details are summarized in Table 2. $\mathrm{H}$ atoms were located in difference Fourier maps and were refined with isotropic displacement parameters.

\section{Figure}

$\mathrm{R}, \mathrm{R}$ - and an S,S-configured diole are connected via short hydrogen bonds $\left[\mathrm{O} 1-\mathrm{H} 1 \mathrm{O} \cdots \mathrm{O} 2^{\mathrm{ii}}=2.725(3) \AA\right]$ to a centrosymmetric dimer (Table 1$)$. A $\mathrm{C}-\mathrm{H} \cdots \mathrm{O}$ hydrogen bond $\left[\mathrm{C} 5-\mathrm{H} 5 \cdots \mathrm{O} 1^{\mathrm{i}}=3.297\right.$ (4) $\AA$ ] forms a chain parallel to the $\mathrm{b}$ axis. Hydrogen bonds between atoms $\mathrm{O} 1$ and $\mathrm{O} 2$ to the water molecule $\left[\mathrm{O} 2-\mathrm{H} 2 \mathrm{O} \cdots \mathrm{O} 3^{\mathrm{i}}=2.773(3) \AA\right.$ and $\mathrm{O} 3-\mathrm{H} 3 \mathrm{O} \cdots \mathrm{O} 1=$ 2.999 (3) $\AA$ ] connect these chains into layers in the ab plane.

\section{Synthesis and crystallization}

The title compound was prepared from ethyl magnesium bromide, acetylene and chloral according to Gorgues et al. (1986) and Dupont (1910) followed by aqueous work-up. A mixture of three stereoisomers was obtained. Recrystallization from ethanol solution gave the title compound. ${ }^{1} \mathrm{H}$ NMR: $\left(\mathrm{CDCl}_{3} / \mathrm{DMSO}-d_{6}, 400 \mathrm{MHz}\right): \delta 7.05(2 \mathrm{H}, \mathrm{OH}), 4.79(2 \mathrm{H}, \mathrm{CH}$,

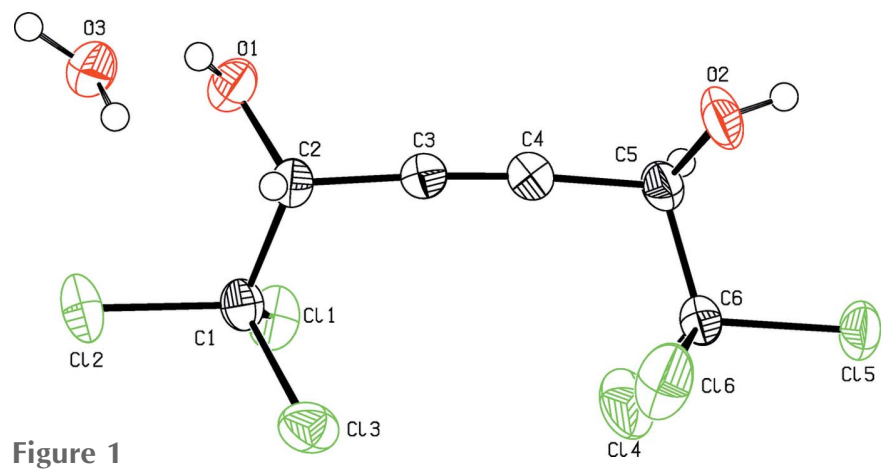

View of the title compound. Displacement ellipsoids are drawn at the $50 \%$ probability level.
Table 2

Experimental details.

\begin{tabular}{|c|c|}
\hline \multicolumn{2}{|l|}{ Crystal data } \\
\hline Chemical formula & $2 \mathrm{C}_{6} \mathrm{H}_{4} \mathrm{Cl}_{6} \mathrm{O}_{2} \cdot \mathrm{H}_{2} \mathrm{O}$ \\
\hline$M_{\mathrm{r}}$ & 659.60 \\
\hline Crystal system, space group & Monoclinic, $I 2 / a$ \\
\hline Temperature $(\mathrm{K})$ & 193 \\
\hline$a, b, c(\AA)$ & $\begin{array}{l}19.8354(11), 5.8480(2), \\
\quad 21.7082(13)\end{array}$ \\
\hline$\beta\left({ }^{\circ}\right)$ & $108.321(4)$ \\
\hline$V\left(\AA^{3}\right)$ & $2390.5(2)$ \\
\hline$Z$ & 4 \\
\hline Radiation type & Мо $K \alpha$ \\
\hline$\mu\left(\mathrm{mm}^{-1}\right)$ & 1.41 \\
\hline Crystal size $(\mathrm{mm})$ & $0.39 \times 0.07 \times 0.06$ \\
\hline \multicolumn{2}{|l|}{ Data collection } \\
\hline Diffractometer & Stoe IPDS $2 \mathrm{~T}$ \\
\hline Absorption correction & $\begin{array}{l}\text { Integration ( } X \text {-RED32; Stoe \& Cie, } \\
\quad 2006 b)\end{array}$ \\
\hline$T_{\min }, T_{\max }$ & $0.714,0.933$ \\
\hline $\begin{array}{l}\text { No. of measured, independent and } \\
\text { observed }[I>2 \sigma(I)] \text { reflections }\end{array}$ & $6305,2965,2202$ \\
\hline$R_{\text {int }}$ & 0.026 \\
\hline$(\sin \theta / \lambda)_{\max }\left(\AA^{-1}\right)$ & 0.668 \\
\hline \multicolumn{2}{|l|}{ Refinement } \\
\hline$R\left[F^{2}>2 \sigma\left(F^{2}\right)\right], w R\left(F^{2}\right), S$ & $0.039,0.090,1.03$ \\
\hline No. of reflections & 2965 \\
\hline No. of parameters & 152 \\
\hline $\mathrm{H}$-atom treatment & All $\mathrm{H}$-atom parameters refined \\
\hline$\Delta \rho_{\max }, \Delta \rho_{\min }\left(\mathrm{e} \AA^{-3}\right)$ & $0.59,-0.57$ \\
\hline
\end{tabular}

Computer programs: X-AREA (Stoe \& Cie, 2006a), X-RED32 (Stoe \& Cie, 2006b), SIR2004 (Altomare et al., 1999) and SHELXL2014 (Sheldrick, 2015).

$\left.{ }^{1} J_{\mathrm{CH}}=154 \mathrm{~Hz}\right)$. Recrystallization from chloroform solution yielded colourless crystals (m.p. $408 \mathrm{~K}$ ).

\section{Refinement}

Crystal data, data collection and structure refinement details are summarized in Table $2 . \mathrm{H}$ atoms were located in difference

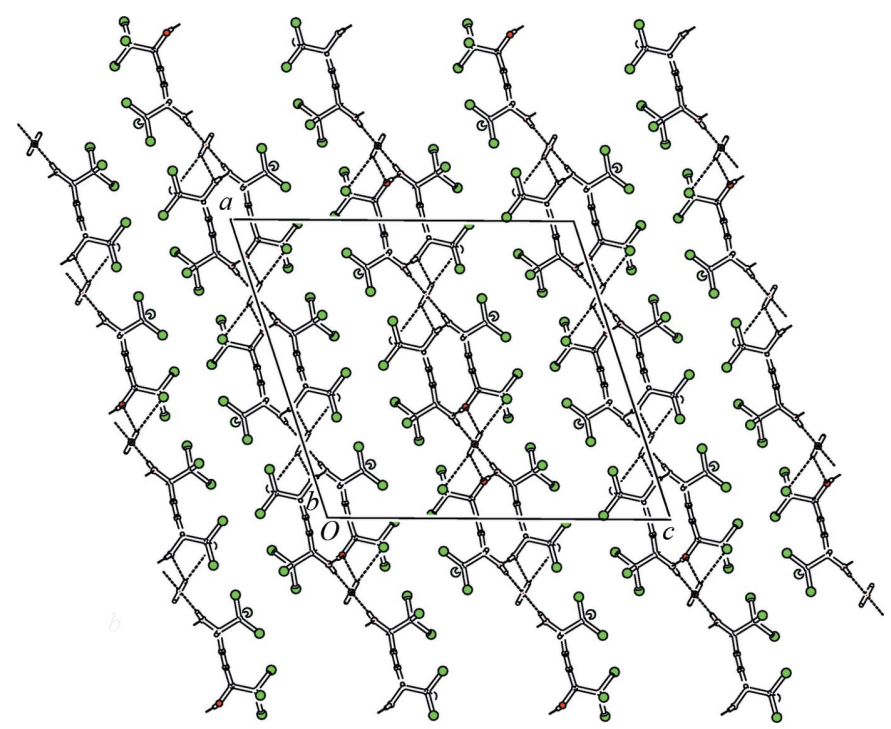

Figure 2

Part of the packing diagram, viewed along the $b$ axis. Hydrogen bonds are shown with dashed lines. 


\section{full crystallographic data}

IUCrData (2017). 2, x171236 [https://doi.org/10.1107/S2414314617012366]

rac-1, 1,1, 6,6,6-Hexachlorohex-3-yne-2,5-diol hemihydrate

Heiner Detert and Dieter Schollmeyer

rac-1,1,1,6,6,6-Hexachlorohex-3-yne-2,5-diol hemihydrate

Crystal data

$2 \mathrm{C}_{6} \mathrm{H}_{4} \mathrm{Cl}_{6} \mathrm{O}_{2} \cdot \mathrm{H}_{2} \mathrm{O}$

$M_{r}=659.60$

Monoclinic, $I 2 / a$

$a=19.8354(11) \AA$

$b=5.8480(2) \AA$

$c=21.7082(13) \AA$

$\beta=108.321(4)^{\circ}$

$V=2390.5(2) \AA^{3}$

$Z=4$

$F(000)=1304$

Data collection

Stoe IPDS 2T

diffractometer

Radiation source: sealed X-ray tube, 12 x 0.4

$\mathrm{mm}$ long-fine focus

Detector resolution: 6.67 pixels $\mathrm{mm}^{-1}$

rotation method scans

Absorption correction: integration

(X-RED32; Stoe \& Cie, 2006b)

$T_{\min }=0.714, T_{\max }=0.933$

\section{Refinement}

Refinement on $F^{2}$

Least-squares matrix: full

$R\left[F^{2}>2 \sigma\left(F^{2}\right)\right]=0.039$

$w R\left(F^{2}\right)=0.090$

$S=1.03$

2965 reflections

152 parameters

0 restraints

Special details

Geometry. All esds (except the esd in the dihedral angle between two 1.s. planes) are estimated using the full covariance matrix. The cell esds are taken into account individually in the estimation of esds in distances, angles and torsion angles; correlations between esds in cell parameters are only used when they are defined by crystal symmetry. An approximate (isotropic) treatment of cell esds is used for estimating esds involving l.s. planes.
$D_{\mathrm{x}}=1.833 \mathrm{Mg} \mathrm{m}^{-3}$

Melting point: $408 \mathrm{~K}$

Mo $K \alpha$ radiation, $\lambda=0.71073 \AA$

Cell parameters from 5782 reflections

$\theta=3.4-28.3^{\circ}$

$\mu=1.41 \mathrm{~mm}^{-1}$

$T=193 \mathrm{~K}$

Column, colourless

$0.39 \times 0.07 \times 0.06 \mathrm{~mm}$

6305 measured reflections

2965 independent reflections

2202 reflections with $I>2 \sigma(I)$

$R_{\text {int }}=0.026$

$\theta_{\max }=28.3^{\circ}, \theta_{\min }=2.4^{\circ}$

$h=-26 \rightarrow 26$

$k=-7 \rightarrow 6$

$l=-24 \rightarrow 28$
Hydrogen site location: difference Fourier map

All $\mathrm{H}$-atom parameters refined

$w=1 /\left[\sigma^{2}\left(F_{\mathrm{o}}^{2}\right)+(0.0295 P)^{2}+6.4827 P\right]$

where $P=\left(F_{\mathrm{o}}^{2}+2 F_{\mathrm{c}}^{2}\right) / 3$

$(\Delta / \sigma)_{\max }<0.001$

$\Delta \rho_{\max }=0.59 \mathrm{e} \AA^{-3}$

$\Delta \rho_{\min }=-0.57$ e $\AA^{-3}$ 
Fractional atomic coordinates and isotropic or equivalent isotropic displacement parameters $\left(\AA^{2}\right)$

\begin{tabular}{|c|c|c|c|c|}
\hline & $x$ & $y$ & $z$ & $U_{\text {iso }} * / U_{\text {eq }}$ \\
\hline $\mathrm{C} 1$ & $0.58326(13)$ & $0.4054(5)$ & $0.37414(13)$ & $0.0269(5)$ \\
\hline $\mathrm{C} 2$ & $0.56624(13)$ & $0.4444(5)$ & $0.43828(13)$ & $0.0268(5)$ \\
\hline $\mathrm{H} 2$ & $0.5573(14)$ & $0.608(5)$ & $0.4386(13)$ & $0.026(7)^{*}$ \\
\hline C3 & $0.50278(14)$ & $0.3142(5)$ & $0.43757(12)$ & $0.0296(6)$ \\
\hline $\mathrm{C} 4$ & $0.45026(15)$ & $0.2097(5)$ & $0.43394(13)$ & $0.0314(6)$ \\
\hline $\mathrm{C} 5$ & $0.38356(15)$ & $0.0835(5)$ & $0.42428(13)$ & $0.0303(6)$ \\
\hline H5 & $0.3951(17)$ & $-0.077(6)$ & $0.4347(16)$ & $0.046(9)^{*}$ \\
\hline C6 & $0.34051(13)$ & $0.0815(5)$ & $0.35171(13)$ & $0.0270(5)$ \\
\hline $\mathrm{O} 1$ & $0.62524(11)$ & $0.3777(4)$ & $0.49119(10)$ & $0.0345(5)$ \\
\hline $\mathrm{H} 1 \mathrm{O}$ & $0.637(2)$ & $0.484(7)$ & $0.5082(19)$ & $0.051(12)^{*}$ \\
\hline $\mathrm{O} 2$ & $0.34423(13)$ & $0.1915(5)$ & $0.46020(11)$ & $0.0489(7)$ \\
\hline $\mathrm{H} 2 \mathrm{O}$ & $0.319(2)$ & $0.114(7)$ & $0.4692(19)$ & $0.059(13)^{*}$ \\
\hline $\mathrm{O} 3$ & 0.7500 & $0.0752(6)$ & 0.5000 & $0.0380(7)$ \\
\hline $\mathrm{H} 3 \mathrm{O}$ & $0.717(3)$ & $0.171(10)$ & $0.475(3)$ & $0.12(2)^{*}$ \\
\hline $\mathrm{Cl1}$ & $0.59756(4)$ & $0.11144(12)$ & $0.36352(4)$ & $0.03662(17)$ \\
\hline $\mathrm{Cl} 2$ & $0.66181(4)$ & $0.55802(13)$ & $0.37759(4)$ & 0.04003 (19) \\
\hline $\mathrm{Cl} 3$ & $0.51257(4)$ & $0.50491(14)$ & 0.30818 & $0.04010(19)$ \\
\hline $\mathrm{Cl} 14$ & $0.38978(5)$ & $-0.05417(17)$ & $0.30774(4)$ & $0.0551(3)$ \\
\hline $\mathrm{C} 15$ & $0.26084(4)$ & $-0.07339(13)$ & $0.34107(4)$ & $0.03746(18)$ \\
\hline $\mathrm{Cl} 6$ & $0.31978(4)$ & $0.36244(14)$ & $0.32294(5)$ & $0.0547(2)$ \\
\hline
\end{tabular}

Atomic displacement parameters $\left(\AA^{2}\right)$

\begin{tabular}{lllllll}
\hline & $U^{11}$ & $U^{22}$ & $U^{33}$ & $U^{12}$ & $U^{13}$ & $U^{23}$ \\
\hline C1 & $0.0242(12)$ & $0.0244(12)$ & $0.0341(13)$ & $0.0017(10)$ & $0.0119(10)$ & $0.0002(11)$ \\
C2 & $0.0232(12)$ & $0.0271(14)$ & $0.0300(13)$ & $-0.0016(10)$ & $0.0083(10)$ & $0.0002(11)$ \\
C3 & $0.0268(13)$ & $0.0357(15)$ & $0.0257(12)$ & $-0.0011(11)$ & $0.0075(10)$ & $0.0015(11)$ \\
C4 & $0.0302(14)$ & $0.0358(15)$ & $0.0292(13)$ & $-0.0032(12)$ & $0.0109(11)$ & $0.0016(12)$ \\
C5 & $0.0311(14)$ & $0.0315(15)$ & $0.0312(14)$ & $-0.0084(12)$ & $0.0138(11)$ & $-0.0003(12)$ \\
C6 & $0.0251(12)$ & $0.0271(13)$ & $0.0312(13)$ & $-0.0024(10)$ & $0.0123(10)$ & $-0.0012(11)$ \\
O1 & $0.0297(10)$ & $0.0338(12)$ & $0.0349(11)$ & $-0.0043(9)$ & $0.0028(8)$ & $-0.0005(9)$ \\
O2 & $0.0507(14)$ & $0.0622(16)$ & $0.0484(13)$ & $-0.0332(13)$ & $0.0367(11)$ & $-0.0271(12)$ \\
O3 & $0.0278(15)$ & $0.0455(18)$ & $0.0381(16)$ & 0.000 & $0.0066(12)$ & 0.000 \\
C11 & $0.0322(3)$ & $0.0265(3)$ & $0.0517(4)$ & $0.0022(3)$ & $0.0140(3)$ & $-0.0071(3)$ \\
C12 & $0.0346(4)$ & $0.0347(4)$ & $0.0601(5)$ & $-0.0071(3)$ & $0.0282(3)$ & $-0.0022(3)$ \\
C13 & $0.0384(4)$ & $0.0489(4)$ & $0.0334(3)$ & $0.0119(3)$ & $0.0118(3)$ & $0.0097(3)$ \\
C14 & $0.0544(5)$ & $0.0652(6)$ & $0.0615(5)$ & $-0.0178(4)$ & $0.0408(4)$ & $-0.0298(5)$ \\
C15 & $0.0301(3)$ & $0.0397(4)$ & $0.0436(4)$ & $-0.0134(3)$ & $0.0130(3)$ & $-0.0058(3)$ \\
C16 & $0.0418(4)$ & $0.0358(4)$ & $0.0742(6)$ & $-0.0033(3)$ & $0.0004(4)$ & $0.0202(4)$
\end{tabular}

Geometric parameters $(\AA, \stackrel{\circ}{)})$

\begin{tabular}{llll}
\hline $\mathrm{C} 1-\mathrm{C} 2$ & $1.549(4)$ & $\mathrm{C} 5-\mathrm{O} 2$ & $1.414(3)$ \\
$\mathrm{C} 1-\mathrm{Cl} 3$ & $1.758(3)$ & $\mathrm{C} 5-\mathrm{C} 6$ & $1.538(4)$ \\
$\mathrm{C} 1-\mathrm{C} 11$ & $1.769(3)$ & $\mathrm{C} 5-\mathrm{H} 5$ & $0.98(4)$
\end{tabular}




\begin{tabular}{|c|c|c|c|}
\hline $\mathrm{C} 1-\mathrm{Cl} 2$ & $1.776(3)$ & $\mathrm{C} 6-\mathrm{Cl} 4$ & $1.756(3)$ \\
\hline $\mathrm{C} 2-\mathrm{O} 1$ & $1.413(3)$ & $\mathrm{C} 6-\mathrm{Cl} 6$ & $1.760(3)$ \\
\hline $\mathrm{C} 2-\mathrm{C} 3$ & $1.467(4)$ & $\mathrm{C} 6-\mathrm{Cl} 5$ & $1.772(3)$ \\
\hline $\mathrm{C} 2-\mathrm{H} 2$ & $0.97(3)$ & $\mathrm{O} 1-\mathrm{H} 1 \mathrm{O}$ & $0.72(4)$ \\
\hline $\mathrm{C} 3-\mathrm{C} 4$ & $1.189(4)$ & $\mathrm{O} 2-\mathrm{H} 2 \mathrm{O}$ & $0.75(4)$ \\
\hline $\mathrm{C} 4-\mathrm{C} 5$ & $1.471(4)$ & $\mathrm{O} 3-\mathrm{H} 3 \mathrm{O}$ & $0.90(5)$ \\
\hline $\mathrm{C} 2-\mathrm{C} 1-\mathrm{Cl} 3$ & $109.87(17)$ & $\mathrm{O} 2-\mathrm{C} 5-\mathrm{C} 4$ & $108.9(2)$ \\
\hline $\mathrm{C} 2-\mathrm{C} 1-\mathrm{C} 11$ & $110.43(19)$ & $\mathrm{O} 2-\mathrm{C} 5-\mathrm{C} 6$ & $110.1(2)$ \\
\hline $\mathrm{Cl} 3-\mathrm{C} 1-\mathrm{Cl}$ & $109.56(15)$ & $\mathrm{C} 4-\mathrm{C} 5-\mathrm{C} 6$ & $109.5(2)$ \\
\hline $\mathrm{C} 2-\mathrm{C} 1-\mathrm{Cl} 2$ & $108.92(18)$ & $\mathrm{O} 2-\mathrm{C} 5-\mathrm{H} 5$ & $116(2)$ \\
\hline $\mathrm{Cl} 3-\mathrm{C} 1-\mathrm{Cl} 2$ & $109.42(14)$ & $\mathrm{C} 4-\mathrm{C} 5-\mathrm{H} 5$ & $108(2)$ \\
\hline $\mathrm{C} 11-\mathrm{C} 1-\mathrm{Cl} 2$ & $108.62(14)$ & $\mathrm{C} 6-\mathrm{C} 5-\mathrm{H} 5$ & $104(2)$ \\
\hline $\mathrm{O} 1-\mathrm{C} 2-\mathrm{C} 3$ & $110.7(2)$ & $\mathrm{C} 5-\mathrm{C} 6-\mathrm{Cl} 4$ & $109.60(19)$ \\
\hline $\mathrm{O} 1-\mathrm{C} 2-\mathrm{C} 1$ & $109.4(2)$ & $\mathrm{C} 5-\mathrm{C} 6-\mathrm{Cl} 6$ & $110.4(2)$ \\
\hline $\mathrm{C} 3-\mathrm{C} 2-\mathrm{C} 1$ & $110.1(2)$ & $\mathrm{Cl} 4-\mathrm{C} 6-\mathrm{Cl} 6$ & $109.69(15)$ \\
\hline $\mathrm{O} 1-\mathrm{C} 2-\mathrm{H} 2$ & $111.8(17)$ & $\mathrm{C} 5-\mathrm{C} 6-\mathrm{Cl} 5$ & $108.81(18)$ \\
\hline $\mathrm{C} 3-\mathrm{C} 2-\mathrm{H} 2$ & $110.6(17)$ & $\mathrm{Cl} 4-\mathrm{C} 6-\mathrm{Cl} 5$ & $108.90(15)$ \\
\hline $\mathrm{C} 1-\mathrm{C} 2-\mathrm{H} 2$ & $104.0(17)$ & $\mathrm{Cl} 6-\mathrm{C} 6-\mathrm{Cl} 5$ & $109.37(14)$ \\
\hline $\mathrm{C} 4-\mathrm{C} 3-\mathrm{C} 2$ & $176.9(3)$ & $\mathrm{C} 2-\mathrm{O} 1-\mathrm{H} 1 \mathrm{O}$ & $104(3)$ \\
\hline $\mathrm{C} 3-\mathrm{C} 4-\mathrm{C} 5$ & $175.8(3)$ & $\mathrm{C} 5-\mathrm{O} 2-\mathrm{H} 2 \mathrm{O}$ & $114(3)$ \\
\hline $\mathrm{Cl} 3-\mathrm{C} 1-\mathrm{C} 2-\mathrm{O} 1$ & $-176.19(18)$ & $\mathrm{O} 2-\mathrm{C} 5-\mathrm{C} 6-\mathrm{Cl} 4$ & $-179.75(18)$ \\
\hline $\mathrm{C} 11-\mathrm{C} 1-\mathrm{C} 2-\mathrm{O} 1$ & $62.8(2)$ & $\mathrm{C} 4-\mathrm{C} 5-\mathrm{C} 6-\mathrm{Cl} 4$ & $60.5(3)$ \\
\hline $\mathrm{Cl} 2-\mathrm{C} 1-\mathrm{C} 2-\mathrm{O} 1$ & $-56.3(3)$ & $\mathrm{O} 2-\mathrm{C} 5-\mathrm{C} 6-\mathrm{Cl} 6$ & $59.3(3)$ \\
\hline $\mathrm{Cl} 3-\mathrm{C} 1-\mathrm{C} 2-\mathrm{C} 3$ & $62.0(3)$ & $\mathrm{C} 4-\mathrm{C} 5-\mathrm{C} 6-\mathrm{Cl} 6$ & $-60.5(3)$ \\
\hline $\mathrm{C} 11-\mathrm{C} 1-\mathrm{C} 2-\mathrm{C} 3$ & $-59.0(3)$ & $\mathrm{O} 2-\mathrm{C} 5-\mathrm{C} 6-\mathrm{Cl} 5$ & $-60.8(3)$ \\
\hline $\mathrm{Cl} 2-\mathrm{C} 1-\mathrm{C} 2-\mathrm{C} 3$ & $-178.21(19)$ & $\mathrm{C} 4-\mathrm{C} 5-\mathrm{C} 6-\mathrm{Cl} 5$ & $179.5(2)$ \\
\hline
\end{tabular}

Hydrogen-bond geometry $\left(\AA,{ }^{\circ}\right)$

\begin{tabular}{lllll}
\hline$D-\mathrm{H} \cdots A$ & $D-\mathrm{H}$ & $\mathrm{H} \cdots A$ & $D \cdots A$ & $D-\mathrm{H} \cdots A$ \\
\hline $\mathrm{C} 5-\mathrm{H} 5 \cdots \mathrm{O} 1^{\mathrm{i}}$ & $0.98(4)$ & $2.50(4)$ & $3.297(4)$ & $139(3)$ \\
$\mathrm{O} 1-\mathrm{H} 1 O \cdots 2^{2 i}$ & $0.72(4)$ & $2.01(4)$ & $2.725(3)$ & $168(4)$ \\
$\mathrm{O} 2-\mathrm{H} 2 O \cdots 3^{\mathrm{i}}$ & $0.75(4)$ & $2.02(4)$ & $2.773(3)$ & $175(4)$ \\
$\mathrm{O} 3-\mathrm{H} 3 O \cdots \mathrm{O} 1$ & $0.90(5)$ & $2.29(5)$ & $2.999(3)$ & $135(5)$ \\
$\mathrm{O} 3-\mathrm{H} 3 O \cdots \mathrm{Cl} 1$ & $0.90(5)$ & $2.83(5)$ & $3.5120(8)$ & $134(4)$ \\
$\mathrm{O} 3-\mathrm{H} 3 O \cdots \mathrm{Cl} 2$ & $0.90(5)$ & $3.06(5)$ & $3.895(3)$ & $155(5)$ \\
\hline
\end{tabular}

Symmetry codes: (i) $-x+1,-y,-z+1$; (ii) $-x+1,-y+1,-z+1$. 